\title{
Photonic Crystal Cavity with a Thin Low-Index Layer for Silicon-Compatible Nanolight Source
}

\author{
Youngsoo Kim, Young Jin Lee, Seokhyeon Hong, Kihwan Moon and Soon-Hong Kwon * \\ Department of Physics, Chung-Ang University, Seoul 06974, Korea; youngsoo.kim94@gmail.com (Y.K.); \\ youngjin.lee.91@gmail.com (Y.J.L.); lechter@naver.com (S.H.); sinbadra@gmail.com (K.M.) \\ * Correspondence: shkwon@cau.ac.kr; Tel.: +82-02-820-5844
}

Received: 3 August 2018; Accepted: 31 August 2018; Published: 4 September 2018

Featured Application: In this paper, we propose a novel nanocavity consisting of silicon and silica with a low optical loss and high confinement factor in silica. The proposed cavity is a good candidate for silicon-compatible nanolight sources such as nanolasers or light-emitting diodes.

\begin{abstract}
The development of an efficient silicon-based nanolight source is an important step for silicon-based photonic integrated circuits. We propose a high quality factor photonic crystal nanocavity consisting of silicon and silica, which can be used as a silicon-compatible nanolight source. We show that this cavity can effectively confine lights in a low-index silica layer with a high confinement factor of 0.25 , in which rare-earth dopants can be embedded as gain materials. The cavity is optimized to have a high quality factor of 15,000 and a mode volume of $0.01 \mathrm{\mu m}^{3}$, while the resonance has a wavelength of $1537 \mathrm{~nm}$. We expect that the high confinement factor in the thin silica layer and the high quality factor of the proposed cavity enable the cavity to be a good candidate for silicon-compatible nanolight sources for use in nanolasers or light-emitting diodes in the telecommunication wavelength region.
\end{abstract}

Keywords: photonic crystals; cavity; Purcell factor; slot mode

\section{Introduction}

Based on well-established complementary metal-oxide semiconductor (CMOS) fabrication technologies, silicon nanophotonic devices have been developed for optical waveguides [1], demultiplexers [2], optical switches, and optical modulators [3,4] because of CMOS compatibility. However, because of the indirect bandgap of silicon, the realization of an efficient light source remains a challenging issue in silicon photonics. Recently, in order to develop a light source for a silicon photonic circuit, several designs, including a III-V semiconductor nanolaser coupled to silicon waveguides $[5,6]$, nanolasers based on active nanomaterials such as nanowires [7], two-dimensional (2D) materials [8], and nanoparticles [9], have been demonstrated by placing other active materials on the silicon photonic circuits. However, in such systems, the coupling efficiencies between the gain materials and the silicon photonic structure become a bottleneck for the efficient device. In contrast, rare-earth atoms, such as erbium (Er) and ytterbium $(\mathrm{Yb})$, and doped silicon oxide or silicon nitride have been reported to have bright telecommunication wavelength emissions [10,11]. Very recently, microdisk cavities of several tens of micrometers demonstrated the enhancement of the emission in the rare-earth doped silicon-compatible materials. However, some papers report a further miniaturized light source with a size of a diffraction limit mode volume, where the small mode size enables ultrafast light modulation [12].

In contrast, photonic crystals, which are periodic structures consisting of two different refractive index materials with a period of a wavelength, are attracting considerable attention in applications of 
nanophotonic devices owing to their ability to control light, confine photons in a wavelength-sized cavity, guide light along the waveguide, and enhance/suppress light emission from emitters in photonic crystals. In particular, the easier fabrication and less-suppressed cavity designs enable the realization of wavelength-sized low-threshold lasers [13], ultralow power consumption optical switches [14], and ultrafast light sources [15]. However, low-index material photonic crystal cavities for rare-earth doped silicon (Si)-based light sources can suffer low $Q$ factors owing to weak total internal reflection (TIR) or large mode volume [16].

In this paper, we propose a transverse magnetic (TM) polarized two-dimensional (2D) photonic crystal thin silica $\left(\mathrm{SiO}_{2}\right)$ layered cavity with a high $Q$ factor, small mode volume $\left(V_{\mathrm{m}}\right)$, and high confinement factor $(\Gamma)$ in the thin $\mathrm{SiO}_{2}$ layer on a square lattice of silicon rods. The proposed cavity is a possible best candidate for the realization of a silicon-compatible nanolight source.

\section{Thin Low-Index Layered Photonic Crystal Cavity Structure}

We propose a photonic crystal $(\mathrm{PhC})$ cavity structure consisting of square lattices of dielectric rods, in which a thin $\mathrm{SiO}_{2}$ layer with thickness of $\mathrm{t}$ is sandwiched between the top and bottom silicon layers, as shown in Figure 1a,b. The total height and radius of each cylinder, and the lattice constant are denoted by $\mathrm{H}, \mathrm{r}$, and a, respectively. The central defect cylinder has radius of $r_{d}$, and the nearest four cylinders are shifted into the center of the cavity with a distance of $d$. The refractive indices are 3.48 and 1.47 for $\mathrm{Si}$ and $\mathrm{SiO}_{2}$, respectively, which are the indices for the telecommunication wavelength of $1550 \mathrm{~nm}$. There is a total of $19 \times 19$ cylinders. For this structure, we calculated the optical properties of the cavity mode and the horizontal $\mathrm{SiO}_{2}$ slot slab waveguide mode, as well as the quality $(Q)$ factor, wavelength, mode volumes, and mode profiles using a home-made three-dimensional (3D) finite-difference time-domain (FDTD) code. Perfectly matched layers are used as boundary conditions for all of the directions in the simulations of the cavity mode, and a periodic boundary condition considering the wavevector is used for the y direction, which is the propagation direction in the simulations of the waveguide mode. A grid of $5 \mathrm{~nm}$ is used. The proposed rod $\mathrm{PhC}$ cavity structure can be fabricated from silicon on insulator substrate using a standard CMOS fabrication process. In addition, by using glutinous substances (e.g., polydimethylsiloxane) [6,17], the whole PhC cavity can be detached from the Si wafer and transferred into other photonic structures such as waveguides.
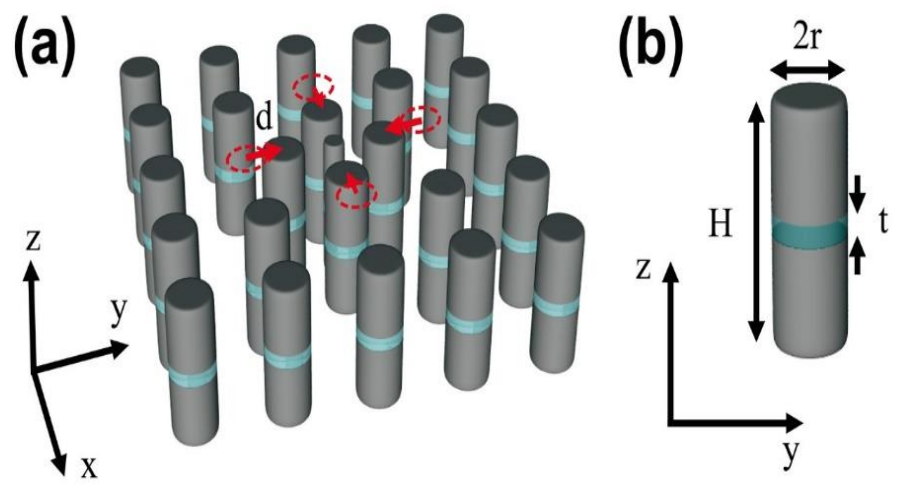

Figure 1. (a) Schematic diagram of the proposed photonic crystal (PhC) cavity consisting of $19 \times 19$ square lattice of cylinders; (b) In each cylinder with a radius of $\mathrm{r}$ and total height of $H$, a thin $\mathrm{SiO}_{2}$ layer, indicated in cyan, with a thickness of $t$ is sandwiched between thick Si layers. The central hole has a smaller radius of $r_{d}$, and the four nearest neighbor rods are shifted into the center over a distance of $d$ from their original positions, as denoted by red dotted circles.

\section{Dispersion Curves of Slot Slab Waveguide}

First, to determine the total height $(H)$ of the cylinder for the strong vertical confinement of lights, we investigated the effect of Si slab height on the effective index. We calculated the dispersion 
curves of a transverse magnetic (TM) Si slab waveguide mode by increasing the height $(H)$ of the slab, as shown in Figure 2a. Here, the dominant electric field is aligned in the height direction. When the height is $200 \mathrm{~nm}$ (red), the dispersion curve follows the light line for small wavevectors and long wavelengths because of weak vertical confinement in the slab. As the height increases, the dispersion curve approaches the dispersion line of an infinitely thick Si slab waveguide (dotted blue line). By using the dispersion curves of Figure 2a, we obtain the effective index of the Si slab waveguide mode as a function of the height $(H)$ for a wavelength of $1550 \mathrm{~nm}$, as shown in Figure 2b. As height increases, the effective index increases, and when height is larger than $800 \mathrm{~nm}$, the index approaches the bulk index of $\mathrm{Si}, 3.48$. Since the effective index of the slab with a height of $1000 \mathrm{~nm}$ is 3.4, which is almost the same value of bulk $\mathrm{Si}$, we chose a total rod height of $1000 \mathrm{~nm}$. In addition, the fabrication of Si rods with a height of $1000 \mathrm{~nm}$ can be accomplished conventionally using standard Si CMOS fabrication technology.
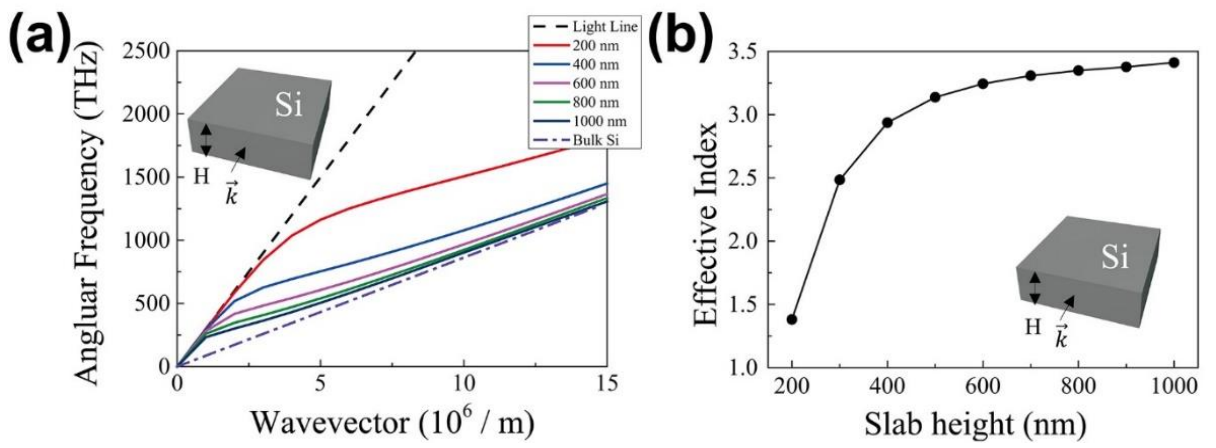

Figure 2. (a) Dispersion curves of a transverse magnetic (TM) silicon (Si) slab waveguide mode for different heights $(H)$ of the slab, ranging from $200 \mathrm{~nm}$ to $1000 \mathrm{~nm}$. The dispersion curve of an infinitely thick Si waveguide mode and light line are indicated by dotted black and blue lines, respectively; (b) Effective index of Si slab waveguide mode as a function of slab height $(H)$ for the wavelength of $1550 \mathrm{~nm}$.

Next, we calculated the effective index of the $\mathrm{SiO}_{2}$ slot waveguide as a function of slot thickness $(t)$, as shown in Figure 3a, where the low-index $\mathrm{SiO}_{2}$ slot is sandwiched between high-index Si materials at the center of the Si slab with a height $(H)$ of $1000 \mathrm{~nm}$. Here, a TM-like waveguide mode with the dominant electric field in the vertical direction to the slab is considered. As the slot thickness increases, the effective index decreases monotonically, because the waveguide mode experiences more low-index material $\left(\mathrm{SiO}_{2}\right)$ than high-index material $(\mathrm{Si})$. For example, the effective indexes in the slots with thicknesses of $10 \mathrm{~nm}$ and $50 \mathrm{~nm}$ are 3.29 and the 3.16, respectively. Figure $3 b$ shows the strong confinement of the z-directional electric field intensity profiles for the waveguides of the $10-\mathrm{nm}$ and $50-\mathrm{nm}$ thick slots, where the total height is fixed at $1000 \mathrm{~nm}$. The electric field is more strongly confined within the 10-nm thick slot than in the slot thickness of the 50-nm thick slot.

In the electric field intensity line graphs of Figure $3 c$ for different slot thickness, the high electric field intensity is strongly confined in the slot region, as shown in the mode profiles of Figure $3 \mathrm{~b}$. In addition, the 10-nm thick slot shows the highest electric field intensity in the slot, and the peak intensity decreases with increasing slot thickness. Here, the electric field intensity is normalized by the total electric field energy of the waveguide mode for each slot structure. The electric field intensity is related to enhance the emission of the rare earth emitter embedded in the low-index material by the Purcell effect.

In addition to the electric field intensity, the confinement factor denoted by Equation (1) is also considered for the proposed structure as a candidate nanolaser, because the confinement factor can yield an effective modal gain by Equation (2). Therefore, we calculated the confinement factor by 
estimating the ratio of the entire electric field density and slot area density. This confinement factor represents the density of the fields in the slot area $[18,19]$.

$$
\begin{gathered}
\Gamma_{\mathrm{z}}=\frac{\int_{\text {slot }}|E|^{2} d V}{\int|E|^{2} d V}=\frac{\int_{\text {slot }}\left|E_{z}\right|^{2} d V}{\int\left|E_{z}\right|^{2} d V} \\
g_{\text {mod }} \approx \Gamma_{z} g_{i}
\end{gathered}
$$

where $g_{\text {mod }}$ and $g_{i}$ are the modal gain and bulk material gain, respectively.

Figure $3 \mathrm{~d}$ shows the confinement factor (black) and the normalized confinement factor (blue) as functions of horizontal slot thickness. The maximum confinement factor of 0.25 is found at the slot thickness $20 \mathrm{~nm}$. However, the normalized confinement factor, which is defined as the ratio of the confinement factor and the slot thickness, is a maximum at a slot thickness of $10 \mathrm{~nm}$. The normalized confinement factor means the averaged electric field intensity over the slot, where the confinement factors are normalized by the whole electric field. In general, because the rare-earth emitter is randomly distributed over the low-index layer, the normalized confinement factor can guarantee better performance as a light-emitting diode for the suggested structure. Depending on the applications, the structure that has a high confinement factor or normalized confinement factor can be chosen. In the rest of this paper, we use a horizontal slot thickness of $10 \mathrm{~nm}$ at a fixed rod height of $1000 \mathrm{~nm}$ for the high confinement of the cavity mode in the horizontal slot, which will be used as gain materials, including rare-earth dopants.
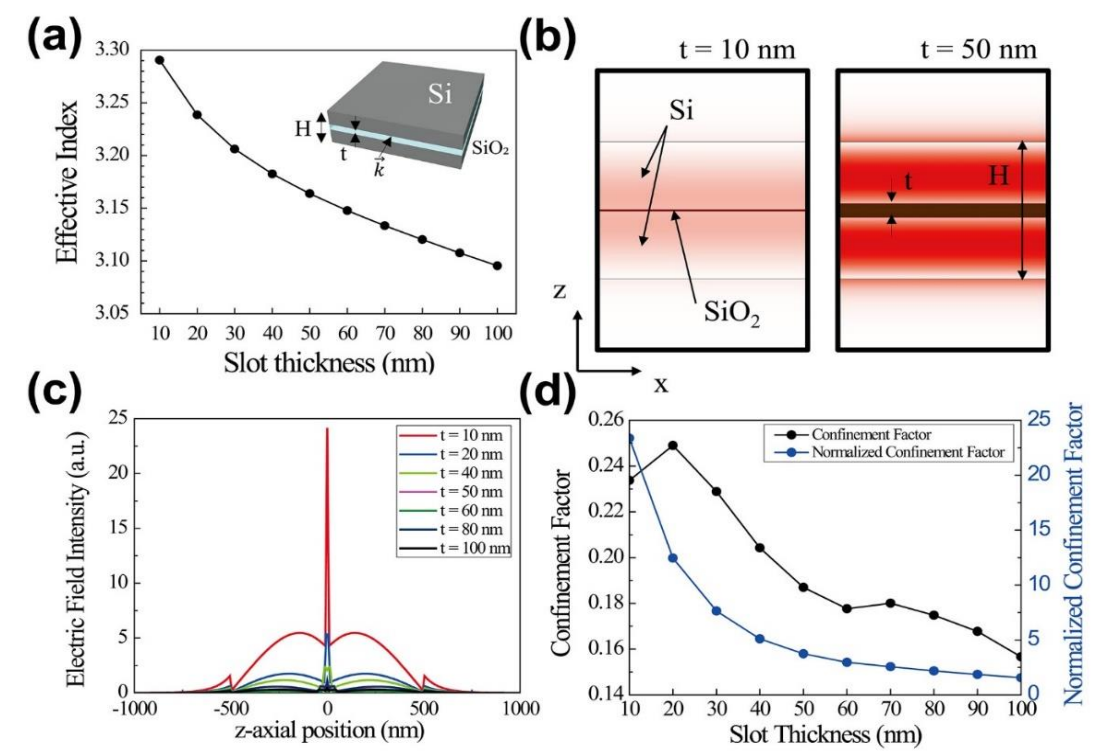

Figure 3. (a) Effective index of a thin $\mathrm{SiO}_{2}$ layer embedded in a Si slab with a height $(H)$ of $1000 \mathrm{~nm}$ as a function of the $\mathrm{SiO}_{2}$ layer thickness. Here, $1550 \mathrm{~nm}$ is considered as the target wavelength; (b) Mode profiles of z-directional electric field intensity for the $\mathrm{SiO}_{2}$ layer thickness ( $t$ ) of $10 \mathrm{~nm}$ (left) and $50 \mathrm{~nm}$ (right); (c) Electric field intensity profiles for different $\mathrm{SiO}_{2}$ layer thicknesses; (d) Confinement factor (black) and normalized confinement factor (blue) as functions of thin $\mathrm{SiO}_{2}$ layer thickness.

\section{Design of Photonic Crystal Cavity Mode}

We designed a 2D photonic crystal by placing cylinders with square lattices consisting of $\mathrm{Si}$ and a thin $\mathrm{SiO}_{2}$ layer, where the total height $(H)$ and $\mathrm{SiO}_{2}$ thickness $(t)$ of each cylinder were $1000 \mathrm{~nm}$ and $10 \mathrm{~nm}$, respectively. The lattice constant $(a)$ and radius $(r)$ of the cylinder are set to $500 \mathrm{~nm}$ and $125 \mathrm{~nm}$, respectively, to achieve a maximum photonic bandgap based on the 3D plane wave expansion (PWE) method. In the calculations of the bandgap, we used a superlattice of the stacked structure 
of air and a 2D rod-type photonic crystal layer. The photonic band diagram of Figure 4a shows that the target wavelength of $1550 \mathrm{~nm}(1216 \mathrm{THz})$ is positioned in the photonic bandgap of the proposed structure. The photonic band diagram was obtained by the 3D PWE method. The $Q$ factor and electric field distributions of a cavity mode were obtained by using FDTD analysis. To induce a cavity mode, we reduced the radius of a cylinder at the center and obtained a fundamental cavity mode at wavelengths of $1549 \mathrm{~nm}, 1568 \mathrm{~nm}$, and $1612 \mathrm{~nm}$ when the defect radius $\left(r_{d}\right)$ was $80 \mathrm{~nm}, 85 \mathrm{~nm}$, and $90 \mathrm{~nm}$, respectively (Figure $4 \mathrm{~b}-\mathrm{d}$ ). The highest quality factor ( $Q$ factor) is 4900 in the structure, with an $r_{d}$ of $90 \mathrm{~nm}$.

(a)

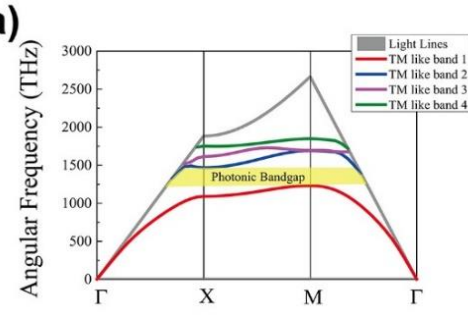

(b)

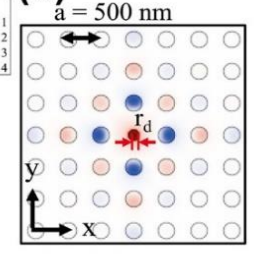

(c)

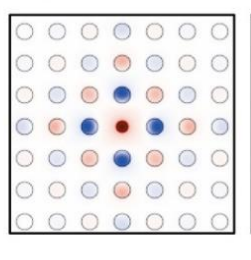

(d)

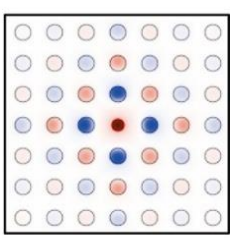

Figure 4. (a) Photonic band diagram of the proposed structure for a cylinder height $(H)$ of $1000 \mathrm{~nm}$, thin $\mathrm{SiO}_{2}$ layer thickness $(t)$ of $10 \mathrm{~nm}$, and cylinder radius $(r)$ of $125 \mathrm{~nm} ;(\mathbf{b}-\mathbf{d})$ z-component electric field mode profiles of cavity modes with different defect radius $\left(r_{d}\right) ;(\mathbf{b}) r_{d}=80 \mathrm{~nm}$; resonant wavelength $1549 \mathrm{~nm}$; total quality factor $=670 ;$ (c) $r_{d}=85 \mathrm{~nm}$; wavelength $=1568 \mathrm{~nm}$; total quality factor $=1000$; (d) $r_{d}=90 \mathrm{~nm}$; wavelength $1612 \mathrm{~nm}$; total quality factor $=4900$. The mode profiles are obtained at the vertical center of the cylinder.

To increase the $Q$ factor further, we suppressed the vertical radiation loss of the cavity by shifting the four nearest neighborhood cylinders into the center defect and changing the mode profiles slightly [20], as shown in Figure 5a. As the nearest cylinders shift into the center and the distance changes from $10 \mathrm{~nm}$ to $100 \mathrm{~nm}$, the resonant wavelength of the cavity mode blue-shifts $1568 \mathrm{~nm}$ to $1509 \mathrm{~nm}$ because of the decrease in the mode volume, as shown in Figure $5 \mathrm{c}$. In contrast, the $Q$ factor has the highest value of 15,000 when $r_{d}$ and the shifted distance (d) are $90 \mathrm{~nm}$ and $60 \mathrm{~nm}$, respectively, and the corresponding wavelength is $1537 \mathrm{~nm}$. Figure $5 \mathrm{~b}$ shows the horizontal and vertical mode profiles in the cavity with $r_{d}$ $=90 \mathrm{~nm}$ and $d=60 \mathrm{~nm}$. The mode is horizontally well confined in the defect cylinder by the photonic bandgap. As expected from the slot waveguide mode profile of Figure 3b, the electric field is strongly confined in the low-index thin $\mathrm{SiO}_{2}$ layer, as shown in the inset of Figure $5 \mathrm{~b}$.

(a)

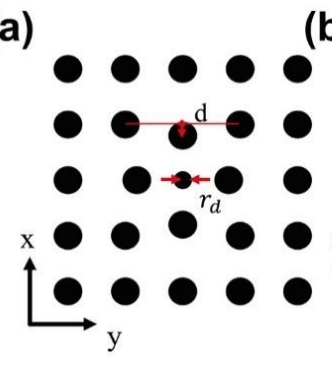

(b) $a=500 \mathrm{~nm}$

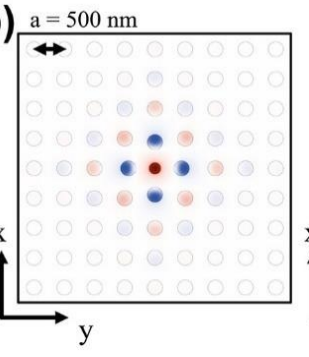

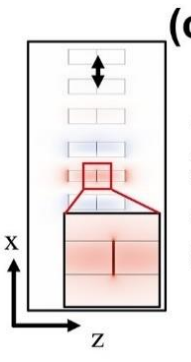

(c)

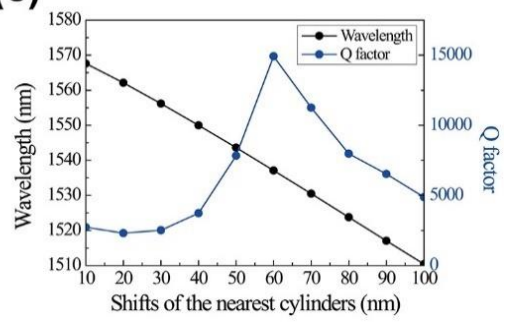

Figure 5. (a) Schematic of the modified cavity with a central defect cylinder and our shifted nearest neighbor cylinders. The four cylinders are shifted into the center over a distance of d; (b) XY and XZ mode profiles of the cavity mode when the defect cylinder radius $r_{d}=90 \mathrm{~nm}$, the shift of the four nearest cylinders $d=60 \mathrm{~nm}$, and the resonant wavelength is $1537 \mathrm{~nm}$. In the XZ mode profile, the inset shows the electric field confinement in the low-index $\mathrm{SiO}_{2}$ layer of the defect cylinder; (c) Resonant wavelengths (black) and $Q$ factors (blue) as functions of the shifted distance of four nearest cylinders while the center defect radius is fixed at $90 \mathrm{~nm}$. The highest $Q$ factor of 15,000 is obtained when the nearest cylinders shifted to the inner side over a distance of $60 \mathrm{~nm}$. 
Based on the optimized cavity structure, we investigated the emission enhancement of rare-earth dopants that can be embedded in the thin $\mathrm{SiO}_{2}$ layer by calculating the Purcell factor. The magnitude of the enhancement is given by the following Purcell factor $F_{P}$ when the emitter is placed at the intensity maximum of the mode profile [21], which corresponds to the proposed cavity. The dopant emitter is placed at the 10-nm thick $\mathrm{SiO}_{2}$ layer of the center cylinder, in which the spatial overlap between the cavity mode and the emitter can be achieved:

$$
F_{P}=\frac{3}{4 \pi^{2}}\left(\frac{\lambda_{c}}{n_{\mathrm{SiO}_{2}}}\right)^{3}\left(\frac{Q}{V}\right)
$$

where $\lambda_{c}$ is the resonant wavelength of the cavity, $n_{\mathrm{SiO}_{2}}$ is the refractive index of silica, $V$ is the mode volume, and $Q$ is the $Q$ factor.

To calculate the Purcell factor, we need to know the mode volume of the cavity mode. The mode volume can be calculated by under the following formula [13]:

$$
V_{\mathrm{mod}}^{\mathrm{eff}}=\frac{\int_{V} \epsilon(\vec{r})|\vec{E}(\vec{r})|^{2} d^{3} r}{\max \left[\epsilon(\vec{r})|\vec{E}(\vec{r})|^{2}\right]}
$$

where $\epsilon(\vec{r})$ is the permittivity as a function of the radial direction, and $\vec{E}(\vec{r})$ is the electric field of the cavity mode as a function of the radial direction at the cavity center.

On the other hand, in case the maximum electric field is found in a low-index region, the mode volume can be represented as a normalized unitless effective mode volume [22]. The normalized mode volume is defined as follows:

$$
\begin{gathered}
\widetilde{V}_{\text {mod }}^{\text {eff }}=\frac{\int_{V} \epsilon(\vec{r})|\vec{E}(\vec{r})|^{2} d^{3} r}{\epsilon\left(\vec{r}_{\text {max }}\right) \max \left[|\vec{E}(\vec{r})|^{2}\right]}\left(\frac{2 n\left(\vec{r}_{\text {max }}\right)}{\lambda}\right)^{3} \\
\widetilde{V}_{\text {mod }}^{\text {eff }}=V_{\text {eff }}\left(\frac{2 n\left(\vec{r}_{\text {max }}\right)}{\lambda}\right)^{3}
\end{gathered}
$$

where $\vec{r}_{\text {max }}$ is the position of the maximum electric field. Therefore, $n\left(\vec{r}_{\max }\right)$ is $n_{\mathrm{SiO}_{2}}=1.48 . \epsilon\left(\vec{r}_{\max }\right)$ is the maximum permittivity of the radial direction. The normalized unitless effective mode volume is 0.64 .

For the optimized cavity structure with $r_{d}=90 \mathrm{~nm}$ and $d=60 \mathrm{~nm}$, the mode volume is obtained as $V_{\mathrm{mod}}^{\text {eff }}=0.01 \mu \mathrm{m}^{3}\left(=0.0089\left(\frac{\lambda_{c}}{n_{\mathrm{SiO}_{2}}}\right)^{3} \mu \mathrm{m}^{3}\right)$, where, $\lambda_{c}$ is $1537 \mathrm{~nm}$ and $n_{\mathrm{SiO}_{2}}$ is 1.48 . By using a wavelength of $1537 \mathrm{~nm}, Q$ factor of 15,000 , and refractive index of $\mathrm{SiO}_{2}$ of 1.48 , the Purcell factor $F_{P}$ is 127,000 . The large Purcell factor means that the proposed cavity structure can enhance the emission of the rare-earth dopants in the low-index layer and will be useful as a silicon-compatible nanolight source.

In order to consider mechanically stable, practical devices in the low-index dielectric substrate, PDMS ( $n=1.396), \mathrm{SiO}_{2}(n=1.47)$, we calculated the effects of the substrate on the $Q$ factor for the optimized PhC cavity $\left(r=120 \mathrm{~nm}, H=1000 \mathrm{~nm}, t=10 \mathrm{~nm}, r_{d}=90 \mathrm{~nm}, d=60 \mathrm{~nm}\right)$. The $Q$ factor decreases to 750 and 490 for PDMS and $\mathrm{SiO}_{2}$ due to leakage loss into the substrate, respectively. In order to overcome leakage loss, the height of the rod can be increased by increasing the distance between the high-intensity region, $\mathrm{SiO}_{2}$ layer, and substrate. When the height of the rod becomes 
$1400 \mathrm{~nm}$ and the distance from the $\mathrm{SiO}_{2}$ layer to the substrate increases to $895 \mathrm{~nm}$, the $Q$ factor is largely improved to 2200 and 870 for $\mathrm{PDMS}$ and $\mathrm{SiO}_{2}$ substrate, respectively.

\section{Conclusions}

In conclusion, we propose a high- $Q$ photonic crystal cavity with a thin low-index $\mathrm{SiO}_{2}$ layer sandwiched at the center of the silicon cylinders. Owing to the optical property of the TM waveguide slot mode, the electric field is strongly confined at the 10-nm thick low-index layer with a confinement factor of 0.23 , where rare-earth dopants with telecommunication emission wavelengths can be embedded. By constructing photonic crystals with square lattices of silicon cylinders with a height of $1000 \mathrm{~nm}$, lattice constant of $500 \mathrm{~nm}$, cylinder radius of $125 \mathrm{~nm}$, and thin low-index layer thickness of $10 \mathrm{~nm}$, the photonic bandgap, including the telecommunication wavelength, can be built. By decreasing the radius of the center cylinder to $90 \mathrm{~nm}$ and shifting the four nearest cylinders into the center over a distance of $60 \mathrm{~nm}$, we can optimize the cavity mode with a resonant wavelength $\lambda_{c}$ of $1537 \mathrm{~nm}$, high $Q$ factor of 15,000 , and mode volume of $0.01 \mu \mathrm{m}^{3}=0.0089\left(\frac{\lambda_{c}}{n_{\mathrm{SiO}_{2}}}\right)^{3} \mu \mathrm{m}^{3}$. If an emitter is embedded at the low-index layer, a high Purcell factor is estimated as 127,000 , owing to strong electric field localization in the low-index layer. We believe that the design of the proposed cavity based on the silicon cylinder and $\mathrm{SiO}_{2}$ layer will be useful in the development of a silicon-based nanolight source, which is urgently required for the CMOS compatible photonic integrated circuit.

Due to spatial spreading, the ion implants of rare-earth dopants have difficulties applying to the thin $\mathrm{SiO}_{2}$ layer of the proposed rod-type photonic crystal structure. The proposed structure can be fabricated by radio frequency confocal magnetron sputtering, and the rare-earth doped thin $\mathrm{SiO}_{2}$ layer also can be prepared by the co-sputtering of three targets: $\mathrm{Si}, \mathrm{SiO}_{2}$, and the oxides of rare-earth materials [23,24].

Author Contributions: Y.K. performed simulations and wrote the manuscript; Y.J.L., S.H. and K.M. designed structures and performed simulations; S.H.K. supervised all simulations and revised the manuscript.

Funding: This research was funded by National Research Foundation of Korea (NRF) grant funded by the Korean government under Grants NRF-2016R1C1B2007007, and NRF-2013M3C1A3065051.

Conflicts of Interest: The authors declare no conflict of interest.

\section{References}

1. Selvaraja, S.K.; Bogaerts, W.; Dumon, P.; Van Thourhout, D.; Baets, R. Subnanometer Linewidth Uniformity in Silicon Nanophotonic Waveguide Devices Using CMOS Fabrication Technology. IEEE J. Sel. Top. Quantum Electron. 2010, 16, 316-324. [CrossRef]

2. Brouckaert, J.; Bogaerts, W.; Dumon, P.; Van Thourhout, D.; Baets, R. Planar Concave Grating Demultiplexer Fabricated on a Nanophotonic Silicon-on-Insulator Platform. J. Lightwave Technol. 2007, 25, 1269-1275. [CrossRef]

3. Liu, A.; Jones, R.; Liao, L.; Samara-Rubio, D.; Rubin, D.; Cohen, O.; Nicolaescu, R.; Paniccia, M. A high-speed silicon optical modulator based on a metal-oxide-semiconductor capacitor. Nature 2004, 427, 615-618. [CrossRef] [PubMed]

4. Martínez, A.; Blasco, J.; Sanchis, P.; Galán, J.V.; García-Rupérez, J.; Jordana, E.; Martí, J. Ultrafast All-Optical Switching in a Silicon-Nanocrystal-Based Silicon Slot Waveguide at Telecom Wavelengths. Nano Lett. 2010, 10, 1506-1511. [CrossRef] [PubMed]

5. Halioua, Y.; Bazin, A.; Monnier, P.; Karle, T.J.; Roelkens, G.; Sagnes, I.; Raj, R.; Raineri, F. Hybrid III-V semiconductor/silicon nanolaser. Opt. Express 2011, 19, 9221. [CrossRef] [PubMed]

6. Lu, T.-W.; Lin, P.-T.; Lee, P.-T. Photonic crystal horizontally slotted nanobeam cavity for silicon-based nanolasers. Opt. Lett. 2012, 37, 569. [CrossRef] [PubMed]

7. Klimov, V.I.; Mikhailovsky, A.A.; Xu, S.; Malko, A.; Hollingsworth, J.A.; Leatherdale, C.A.; Eisler, H.; Yang, P. Optical gain and stimulated emission in nanocrystal quantum dots. Science 2000, 290, 314-317. [CrossRef] [PubMed] 
8. Kim, K.-H.; Hwang, M.-S.; Kim, H.-R.; Choi, J.-H.; No, Y.-S.; Park, H.-G. Direct observation of exceptional points in coupled photonic-crystal lasers with asymmetric optical gains. Nat. Commun. 2016, 7, 13893. [CrossRef] [PubMed]

9. Noginov, M.A.; Zhu, G.; Belgrave, A.M.; Bakker, R.; Shalaev, V.M.; Narimanov, E.E.; Stout, S.; Herz, E.; Suteewong, T.; Wiesner, U. Demonstration of a spaser-based nanolaser. Nature 2009, 460, 1110-1112. [CrossRef] [PubMed]

10. Kippenberg, T.J.; Kalkman, J.; Polman, A.; Vahala, K.J. Demonstration of an erbium-doped microdisk laser on a silicon chip. Phys. Rev. A 2006, 74, 51802. [CrossRef]

11. Heer, S.; Kömpe, K.; Güdel, H.-U.; Haase, M. Highly Efficient Multicolour Upconversion Emission in Transparent Colloids of Lanthanide-Doped $\mathrm{NaYF}_{4}$ Nanocrystals. Adv. Mater. 2004, 16, 2102-2105. [CrossRef]

12. Painter, O. Two-Dimensional Photonic Band-Gap Defect Mode Laser. Science 1999, 284, 1819-1821. [CrossRef] [PubMed]

13. Park, H.-G.; Kim, S.-H.; Kwon, S.-H.; Ju, Y.-G.; Yang, J.-K.; Baek, J.-H.; Kim, S.-B.; Lee, Y.-H. Electrically driven single-cell photonic crystal laser. Science 2004, 305, 1444-1447. [CrossRef] [PubMed]

14. Watts, M.R.; Trotter, D.C.; Young, R.W.; Lentine, A.L. Ultralow power silicon microdisk modulators and switches. In Proceedings of the 2008 5th IEEE International Conference on Group IV Photonics, Cardiff, UK, 17-19 September 2008; pp. 4-6.

15. Rafailov, E.U.; Cataluna, M.A.; Sibbett, W. Mode-locked quantum-dot lasers. Nat. Photonics 2007, 1, $395-401$. [CrossRef]

16. Vahala, K.J. Optical microcavities. Nature 2003, 424, 839-846. [CrossRef] [PubMed]

17. Choi, J.-H.; No, Y.-S.; So, J.-P.; Lee, J.M.; Kim, K.-H.; Hwang, M.-S.; Kwon, S.-H.; Park, H.-G. A high-resolution strain-gauge nanolaser. Nat. Commun. 2016, 7, 11569. [CrossRef] [PubMed]

18. Malviya, N.; Priye, V. Efficient design of silicon slot waveguide optical modulator. Opto-Electron. Rev. 2017, 25, 285-289. [CrossRef]

19. Visser, T.D.; Blok, H.; Demeulenaere, B.; Lenstra, D. Confinement factors and gain in optical amplifiers. IEEE J. Quantum Electron. 1997, 33, 1763-1766. [CrossRef]

20. Frei, W.R.; Johnson, H.T.; Choquette, K.D. Optimization of a single defect photonic crystal laser cavity. J. Appl. Phys. 2008, 103, 033102. [CrossRef]

21. Gerard, J.-M.; Gayral, B. Strong Purcell effect for InAs quantum boxes in three-dimensional solid-state microcavities. J. Lightwave Technol. 1999, 17, 2089-2095. [CrossRef]

22. Robinson, J.T.; Manolatou, C.; Chen, L.; Lipson, M. Ultrasmall Mode Volumes in Dielectric Optical Microcavities. Phys. Rev. Lett. 2005, 95, 143901. [CrossRef] [PubMed]

23. Galli, M.; Gerace, D.; Politi, A.; Liscidini, M.; Patrini, M.; Andreani, L.C.; Canino, A.; Miritello, M.; Lo Savio, R.; Irrera, A.; et al. Direct evidence of light confinement and emission enhancement in active silicon-on-insulator slot waveguides. Appl. Phys. Lett. 2006, 89, 241114. [CrossRef]

24. Galli, M.; Politi, A.; Belotti, M.; Gerace, D.; Liscidini, M.; Patrini, M.; Andreani, L.C.; Miritello, M.; Irrera, A.; Priolo, F.; et al. Strong enhancement of Er3+ emission at room temperature in silicon-on-insulator photonic crystal waveguides. Appl. Phys. Lett. 2006, 88, 251114. [CrossRef]

(C) 2018 by the authors. Licensee MDPI, Basel, Switzerland. This article is an open access article distributed under the terms and conditions of the Creative Commons Attribution (CC BY) license (http:// creativecommons.org/licenses/by/4.0/). 